\title{
MUROS VERDES PARA LAS CONDICIONES AMBIENTALES DE LA REGIÓN NORDESTE ARGENTINA
}

\section{GREEN WALLS FOR ENVIRONMENTAL CONDITIONS OF THE NORTHEAST ARGENTINA REGION}

\author{
D’ Elia, María del Rosario; Pilar, Claudia y Morán, Rosanna \\ Instituto de Investigaciones Tecnológicas de Diseño Ambiental del Hábitat Humano (ITDAHu) \\ Facultad de Arquitectura y Urbanismo - UNNE, Resistecia, Argentina. \\ charii@hotmail.com; claudiapilar2014@gmail.com; moranrosannag@yahoo.com.ar
}

\begin{abstract}
RESUMEN
Los cerramientos verticales son la mayor superficie de intercambio energético entre el ambiente interior y exterior. El objetivo del trabajo es estudiar los muros verdes para las condiciones ambientales de la Región Nordeste Argentina (NEA) a través de casos construidos en la ciudad de Resistencia teniendo en cuenta las variables: resolución tecnológico - constructiva y desempeño ambiental (confort interior e impacto urbano). La hipótesis es que el bajo uso regional se debe a las dificultades de los profesionales para implementar innovaciones, frente a carencias en el mercado de insumos y mano de obra capacitada y una baja valoración de los comitentes sobre los beneficios estéticos y ambientales que conlleva su aplicación. Se realiza el estudio de casos construidos en la ciudad de Resistencia con un abordaje cuanti - cualitativo. La fase cuantitativa se refiere al análisis de los detalles constructivos y la verificación higrotérmica. La fase cualitativa complementa la anterior, a través de entrevistas a profesionales intervinientes en su ejecución. Los principales resultados son la verificación para condiciones reales de las mejoras en las condiciones higrotérmicas de los cerramientos y el impacto favorable a escala arquitectónica, urbana y social.
\end{abstract}

\begin{abstract}
Vertical enclosures are the largest surface of energy exchange between the indoor and outdoor environment. The objective of the work is to study the green walls for the environmental conditions of the Nordeste Argentina Region (NEA) through cases built in the city of Resistencia taking into account the variables: technological resolution - constructive and performance (interior comfort and urban impact). The hypothesis is that the low regional use is due to the difficulties of professionals to implement innovations, in the face of shortages in the market for inputs and skilled labor and a low valuation of the participants on the aesthetic and environmental benefits that involves its application. The study of cases built in the city of Resistencia is carried out with a large - qualitative approach. The quantitative phase refers to the analysis of construction details and hygrothermal verification. The qualitative phase complements the previous one, through interviews with professionals involved in its execution. The main results are the verification for real conditions of improvements in the hygrothermal conditions of the enclosures and the favorable impact on an architectural, urban and social scale.
\end{abstract}

PALABRAS CLAVES: aislamiento higro-termo-acústico, arquitectura sustentable, experiencias regionales, sustentabilidad visual.

KEY WORDS: hygro-thermo-acoustic insulation, sustainable architecture, regional experiences, visual sustainability. 
Artículo RECIBIDO: 15/07/19 | Artículo ACEPTADO: 10/11/19

\section{INTRODUCCIÓN}

El presente trabajo aborda la problemática de la envolvente edilicia considerando que su adecuado diseño es la principal herramienta para disminuir el consumo energético de los edificios y mejorar su estética incorporando el verde con intenciones de subrayar la sustentabilidad visual.

En los últimos años, como parte de las propuestas de arquitectura sostenible, se ha incluido en los paquetes tecnológicos el material vivo, es decir, plantas de distinto porte que generan "Muros Verdes". En el mundo son numerosas las experiencias realizadas aún en contextos climáticos poco favorables. Dada la baja aplicación de esta estrategia en la Región Nordeste de Argentina (NEA), a pesar de que estarían dadas las condiciones ambientales, su aplicación es poco frecuente. Por ello el presente trabajo pretende abordar la temática analizándola desde el punto de vista tecnológico - constructivo, económico y ambiental (confort interior y beneficios a nivel urbano).

\section{DESARROLLO}

La envolvente de los edificios es la "piel" que protege y separa el interior del exterior, ofrece una expresión estética y protege de los agentes climáticos (frío, calor, lluvia y viento), con el adecuado aislamiento térmico y acústico, visual y de seguridad del edificio (Zurigarín, 2013).

Un jardín vertical consiste en tapizar muros con plantas que pueden crecer en distintos medios de cultivo. Pueden prosperar en un sustrato liviano, con algún tipo de suelo natural, o en fibras sintéticas específicas adosadas a bastidores resistentes y de bajo peso.

Existen numerosos mecanismos que van desde los más tradicionales a los más innovadores. Los tipos de fachadas vegetales, según Navarro Portillo, 2013, pueden ser:

- Tradicionales: se trata de las conocidas enredaderas que se trepan a un muro.

- Doble Piel (cables, enrejados modulares, fachada vegetal invernadero y fachadas deslizantes).

- Sistemas precultivados (paneles vegetados en cajas metálicas, sistemas de paneles en celdas drenantes, gaviones metálicos, caños de PVC).

- Hormigón Vegetal

- Sistemas Hidropónicos

- Sistemas con Sustrato

La incorporación del verde en los edificios y en las ciudades produce múltiples beneficios que pueden analizarse a distintas escalas.

A escala arquitectónica:

- Aislamiento térmico y acústico.

- Mejora de la calidad aire interior.

- Mejora de la estética del edificio.

- Transformación permanente de la fachada en las distintas épocas del año dependiendo de la floración.

- Aumento de la sustentabilidad visual.

A escala urbana:

- Fijación del Dióxido de Carbono (CO2).

- Reducción del efecto isla de calor.

- Aumento de la permeabilidad y retención del agua pluvial.

- Retención de polvo, partículas y sustancias contaminantes. 
- Conservación de la naturaleza y biodiversidad urbana.

A escala social:

- Mejora de la salud y el bienestar de la ciudadanía.

- Beneficios económicos: ahorro en los costos de energía de calefacción y refrigeración.

- Aumento del valor del edificio.

- Ahorros en Impuestos (Navarro Portilla, 2013) en aquellas ciudades que prevean beneficios impositivos.

\section{ESTUDIO DE CASOS LOCALES}

La ciudad de Resistencia, capital de la provincia del Chaco, se encuentra ubicada en la zona bioclimática lb (muy cálida húmeda) de la República Argentina. Durante la época estival presenta valores de temperaturas máximas superiores a $34^{\circ} \mathrm{C}$ y valores medios superiores a $26^{\circ} \mathrm{C}$, con amplitudes térmicas siempre inferiores a $15^{\circ} \mathrm{C}$. El periodo invernal es poco significativo con temperaturas medias durante el mes más frío de superiores a $12^{\circ} \mathrm{C}$ (IRAM 11603). Las condiciones climáticas son favorables para el crecimiento de una vegetación variada.

La aplicación de muros verdes tradicionales es una práctica extendida e incluso en caso de abandono de edificaciones la naturaleza se apodera de las construcciones ociosas.

A pesar de que las condiciones ambientales son favorables y que en el mundo existe una fuerte valoración del verde incorporado a la construcción, en la ciudad sólo se identifican casos aislados de muros verdes planificados como elementos de diseño. A continuación se analizan tres casos identificados:

1. Torre Vista.

2. Consejo de Ciencias Económicas.

3. Centro Biotecnológico Agroforestal.

\section{Caso 1: Torre Vista}

- Ubicación: Salta 389. Resistencia, Chaco

- Autores del Proyecto: Arqs. Martín Bodas, Alejandra Maro, Jorge Castro y Ariela Fernández.

- Autores del muro verde: Marina Ventura y Nicolás Frangioli. Empresa Siempre Verde, diseño de jardines.

- Año: 2012.

En la planta baja del edificio, en un espacio semicubierto orientado al Noreste, se dispone un muro verde de triple altura ( $7 \mathrm{~m}$ de alto), que se vivencia tanto a nivel urbano como desde el interior del edificio (Fig. 1). Este muro limita el exterior con un área de servicio (cocheras y sub estación transformadora) por lo que las prestaciones de transmitancia térmica no resultan especialmente gravitantes. 


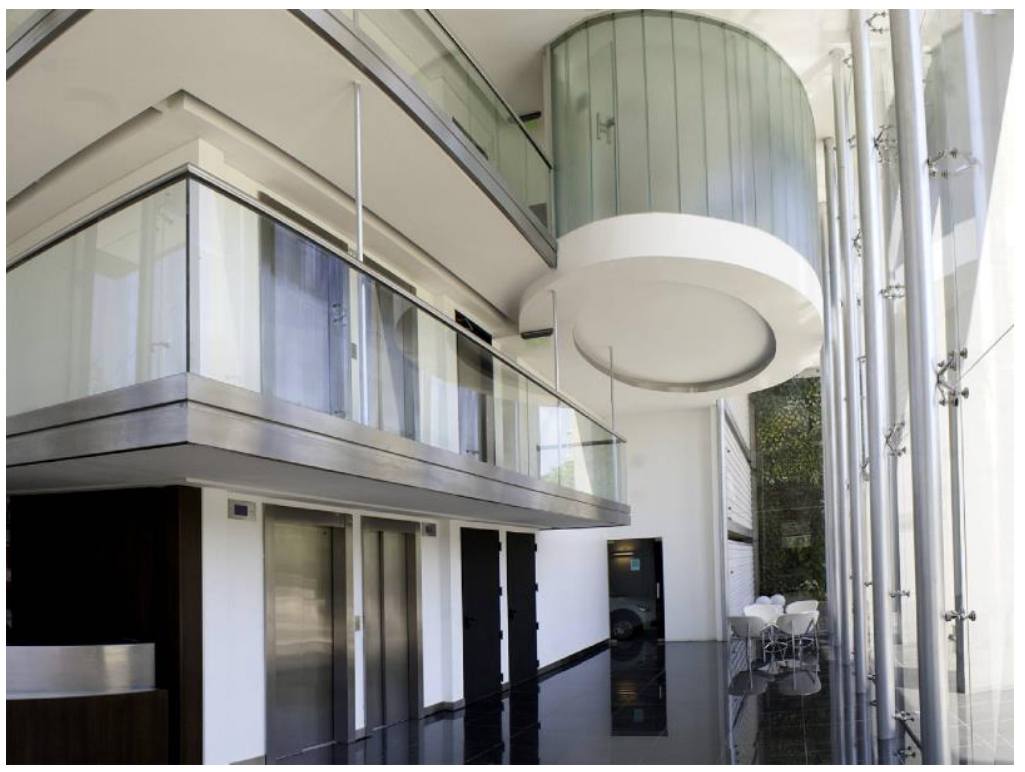

Fig. 1: Vista desde el interior donde se vivencia el muro verde en la Torre Vista.

Fuente: http://www.estudiomarq.com.ar

En cuanto a la construcción del muro verde se trata de un Sistema Hidropónico tecnológicamente resuelto de interior a exterior de la siguiente manera: muro soporte, estructura metálica, placas de PVC, fieltro geotextil reciclado, geotextil con protección ultravioleta y plantas según diseño del jardín. Además posee un sistema de riego automatizado y un sistema de recolección del agua (Fig. 2 y 3 ).
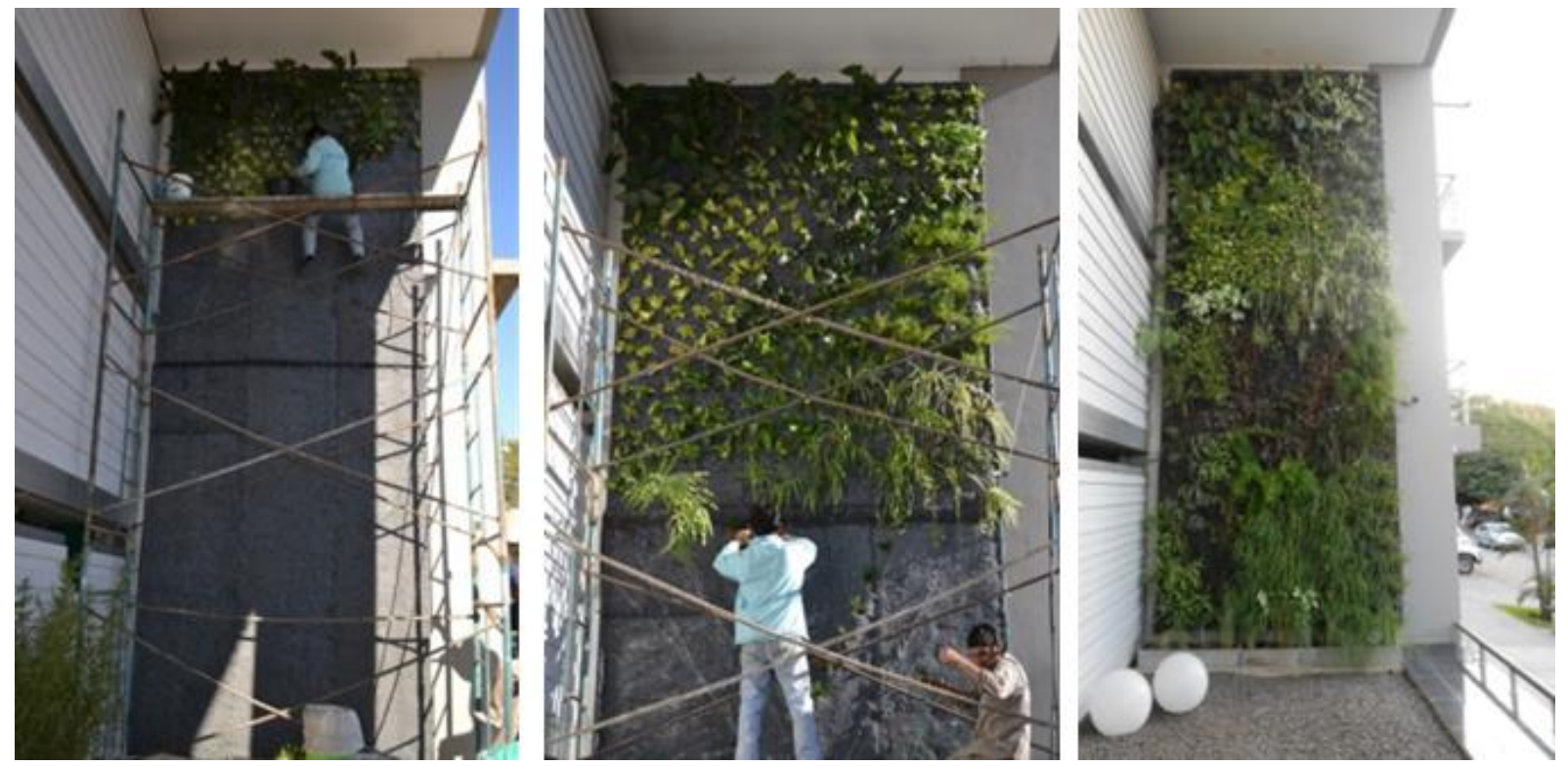

Fig. 2: Proceso de construcción del muro verde.

Fuente: Empresa Siempre Verde, diseño de Jardines. 


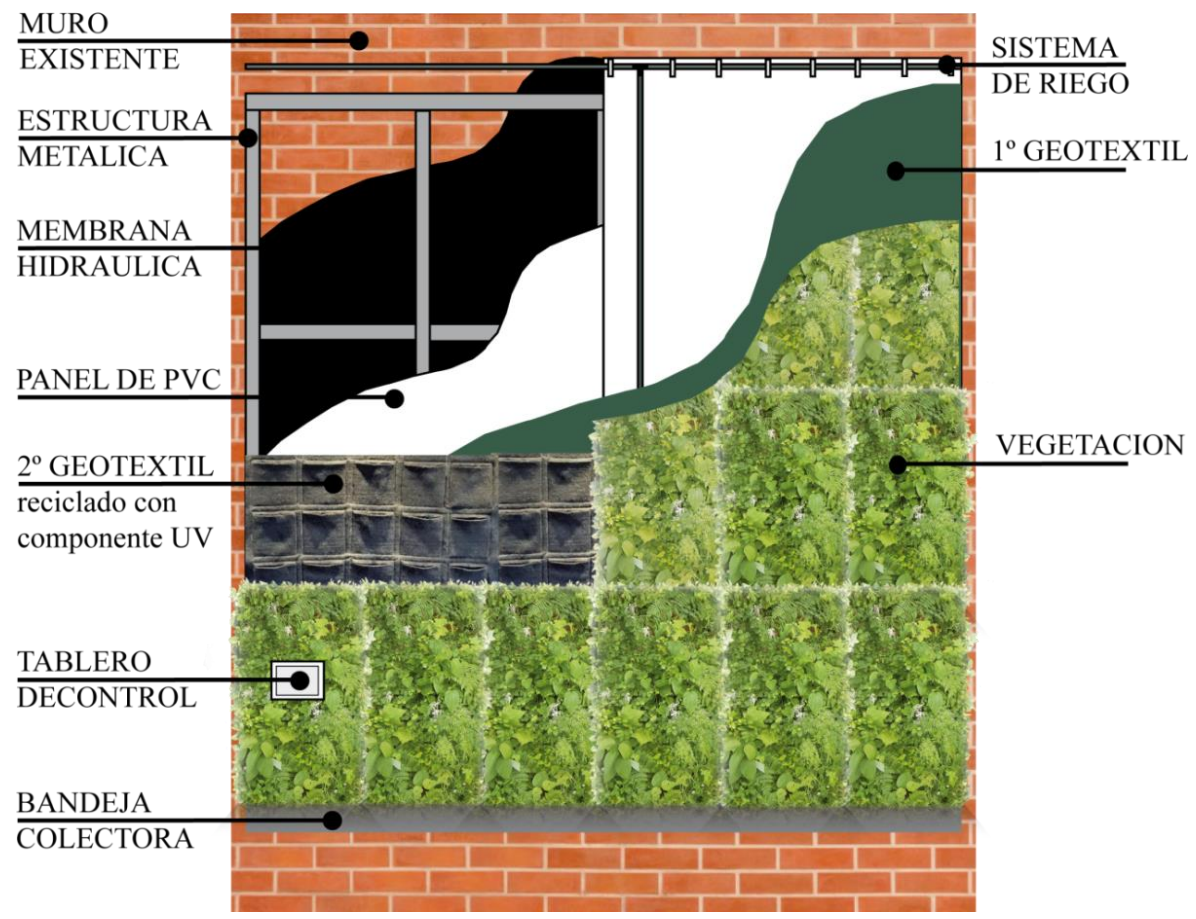

Fig. 3: Despiece tecnológico del jardín hidropónico. Fuente: D' Elia, 2018.

\section{Caso 2: Consejo Profesional de Ciencias Económicas}

- Ubicación: Pellegrini 212. Resistencia. Chaco.

- Autor: Arq. Gustavo Barrios D’Ambra.

- Año: 2015.

Se trata de la ampliación y remodelación de la sede del Consejo Profesional de Ciencias Económicas. El jardín vertical actúa como un tamiz de la radiación solar excesiva de la orientación noroeste de la fachada principal, que por cuestiones corporativas debía presentar una importante proporción de superficie vidriada (Fig. 4).

La fachada del edificio a través de los parasoles verdes, pretende transmitir la "sustentabilidad visual" del conjunto. 


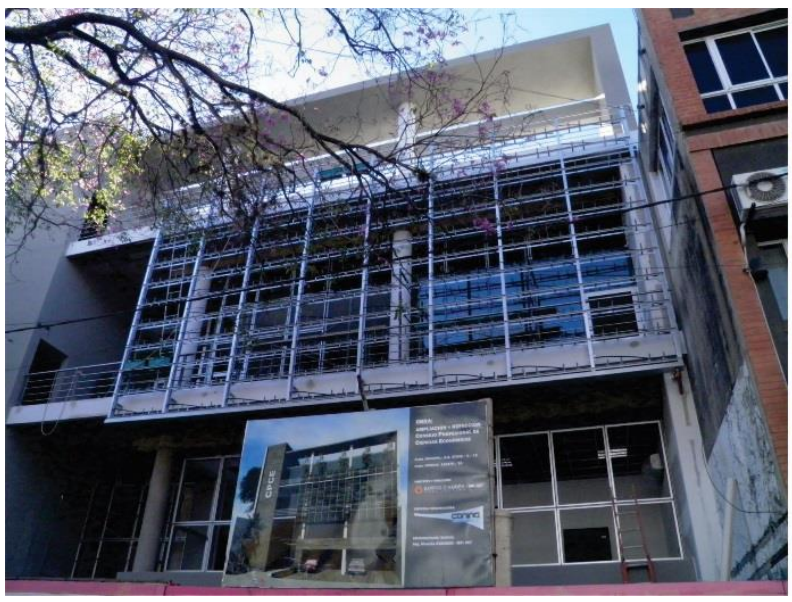

D’ Elia, María del Rosario; Pilar, Claudia y Morán, Rosanna

Fig. 4: Fachada del edificio. A la izquierda durante el proceso constructivo y a la derecha el resultado final. Fuente: Arq. Gustavo Barrios D’Ambra.

El parasolado "verde" corresponde al sector del auditorio, se materializa con jardineras que contienen arbustos (lantana) que requiere bajo mantenimiento, en base a un detallado esqueleto que soporta las macetas y a la vez que permite el pasaje de las instalaciones de riego automatizado.

De esta manera el verde obra de "tamiz" de la excesiva radiación solar de la fachada noroeste, siendo complementada con protecciones horizontales (losa en voladizo de 1,4 $\mathrm{m}$ ) y protecciones verticales interiores (cortinado tipo roller + cortinas tradicionales de pana negra) que busca permitir instancias sucesivas y flexibles de luz u oscuridad según sea el requerimiento y las condiciones meteorológicas. El Sistema Constructivo del muro verde el de doble piel de enrejado modular. Se constituye de una estructura soporte (esqueleto), contenedores con lantanas colocados en una pasarela de mantenimiento y sistema de riego automatizado.

En la Fig. 5 se observa de izquierda a derecha: detalle tecnológico del parasolado verde, en el medio proceso de construcción de la estructura soporte y a la derecha la colocación de las jardineras con arbustos.
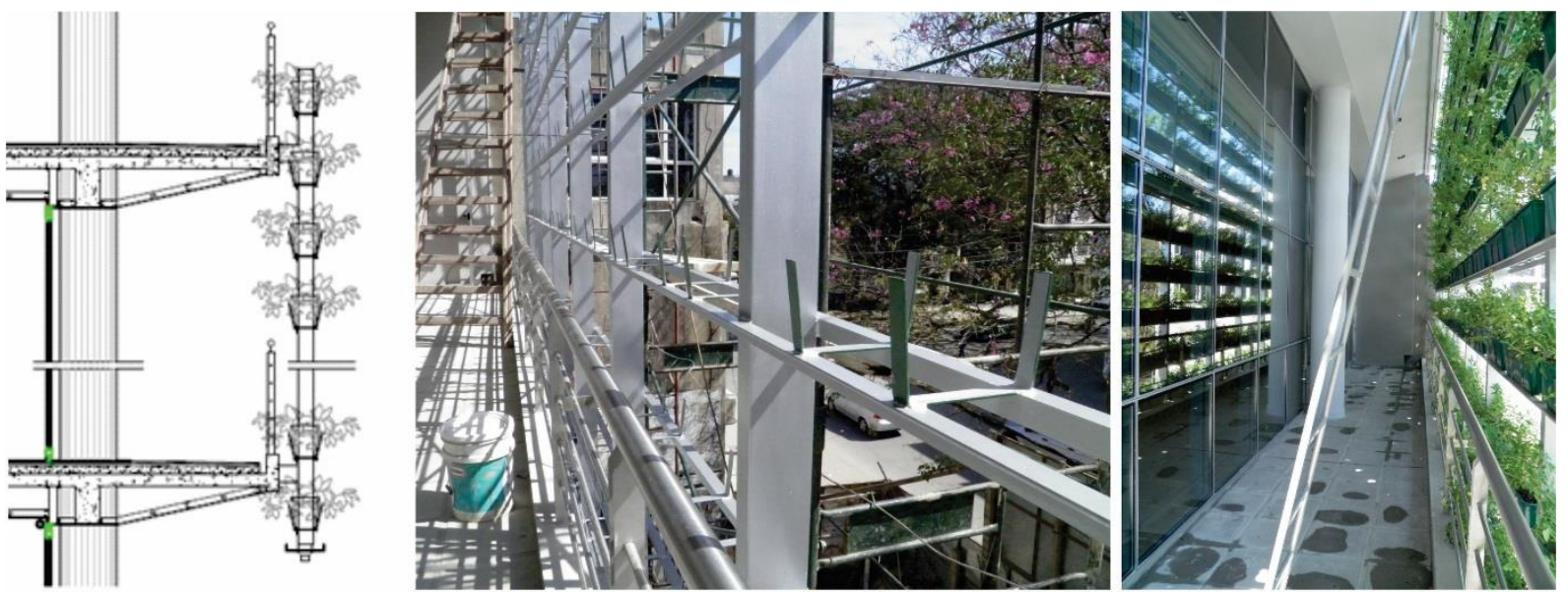

Fig. 5: aspectos tecnológicos del parasol verde. Fuente: Archivos del autor del proyecto, Arq. Gustavo Barrios D'Ambra.

\section{Caso 3: Centro Biotecnológico Agroforestal.}


- Ubicación: Ruta 16, Km. 12,5. Resistencia. Chaco.

- Año: 2014.

Se trata de un edificio destinado a la investigación y desarrollo en biotecnología Agroforestal.

En este edificio es factible identificar dos formas de incorporación de muros verdes: una intervención en el espacio interior, tipo muro verde de sustrato y una exterior de doble piel para protección climática de una galería orientada al noroeste.

El muro verde de interior se expresa como una obra de arte natural de 6 metros de ancho por 2 de alto, a modo de "cuadro vivo". En la Fig. 6 se observa a la izquierda la magnitud de la intervención, en un espacio de doble altura.

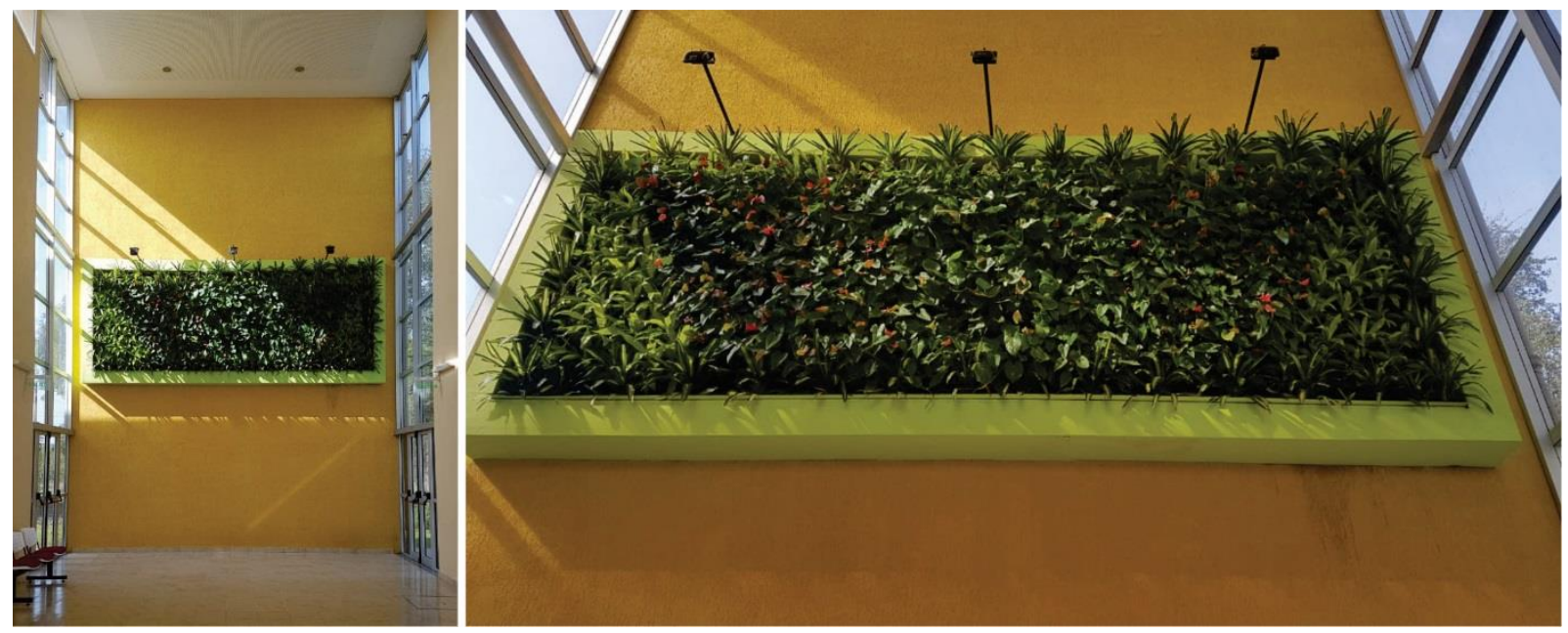

Fig. 6: A la izquierda el cuadro verde en el espacio y a la derecha detalle de vegetación. Fuente: elaboración propia.

De acuerdo a la entrevista realizada a los ejecutores, se trata de una estructura metálica con las correspondientes aislaciones hidráulicas, un sustrato de musgo sphagnum mezclado con perlita. Cuenta con un sistema de riego y un mecanismo de recolección del agua para su reciclado para riego mediante cisterna.

Para cubrir la superficie se utilizaron entre 300 y 360 plantas y se realiza un remplazo periódico de las mismas.

La otra intervención es la piel verde de la galería se trata de un enrejado modular de hierro y malla para que trepen distintas especies como ser Santa Rita, trompeta de bengala (Thunbergia grandiflora) y jazmines.

En la Fig. 7 se observa a la izquierda un detalle tecnológico constructivo de la doble piel, en el medio una fotografía de la espacialidad que le otorga a la galería de doble altura, con orientación noroeste la presencia beneficiosa de la vegetación. A la derecha se observa la vista exterior donde la trompeta de bengala es la que ha tapizado de forma más contundente la estructura. 

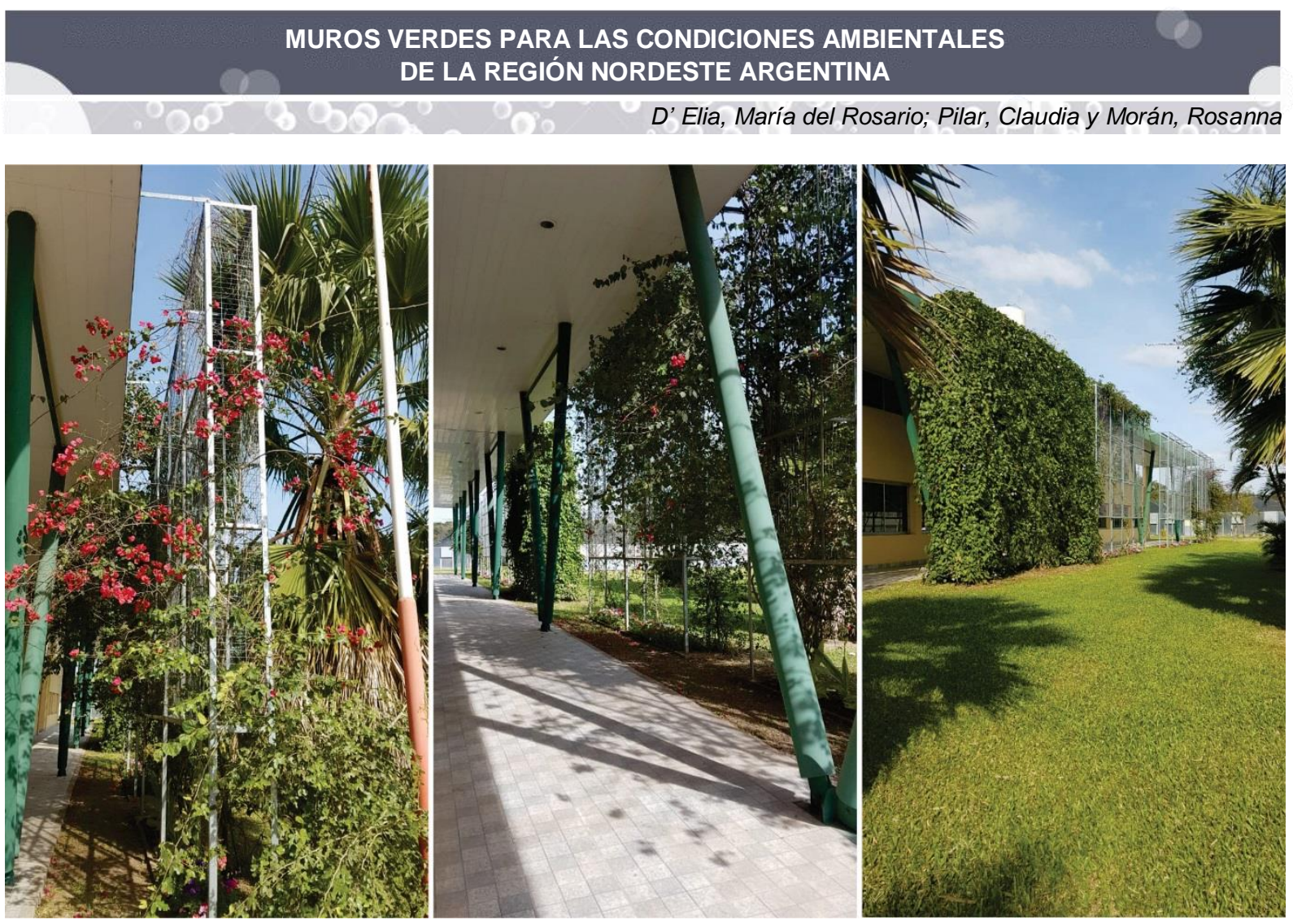

Fig. 7: A la izquierda detalle de la estructura, en el medio, fotografía de la espacialidad de la galería con la piel verde y a la derecha el resultado de la fachada lateral del edificio. Fuente: elaboración propia.

\section{DISCUSIÓN DE RESULTADOS}

A pesar de los pocos casos identificados a nivel local, las tecnologías de ejecución son variadas: hidropónico (caso 1), doble piel de enrejado modular (caso 2), con sustrato (caso 3 interior) y enrejado modular con crecimiento desde el terreno (caso 3 exterior). En todos se verifica una finalidad estética, tendiente a lograr una "sustentabilidad visual".

En el Consejo Profesional (caso 2) y la galería del Centro Biotecnológico (caso 3, exterior) resulta una estrategia de control bioclimático de la radiación solar excesiva (que caracteriza a la zona lb).

Si bien la incorporación del verde aumenta la resistencia térmica del cerramiento, su implementación se fundamenta en la cuestión estética, dado que por ejemplo en el caso 1 se trata del cerramiento que limita el exterior con un sector de servicio. La Arq. Maro, una de las autoras de la obra, manifiesta que el costo de mantenimiento es bajo en relación a la presencia e identidad que le otorga al edificio.

Las tecnologías adoptadas en los casos de estudio podrían ser utilizadas en remodelaciones, rehabilitaciones energéticas o recualificación estéticas de edificios construidos. Esta parece ser una faceta interesante para reverdecer las ciudades sin requerir espacio adicional, muchas veces inexistentes.

\section{CONCLUSIONES}

Las envolventes verticales de los edificios son definitorias en la estética de la construcción y tienen la posibilidad de comunicar una intención de diseño sustentable.

El aumento del compromiso y la responsabilidad ambiental de usuarios, ciudadanos y diseñadores genera una revalorización del verde como síntesis de un anhelo compartido: lograr que la arquitectura y el urbanismo sean más amigables con el ambiente. 
Los muros verdes pueden ser incorporados en variedad de situaciones interiores, exteriores y parasoles, tanto en construcciones nuevas como en remodelaciones. Por el momento resultan aún onerosos, no existen insumos en el mercado local, ni incentivos impositivos en la provincia o la ciudad. Resulta una inversión voluntaria, generalmente asociada a un "marketing ambiental" que repercute favorablemente en el valor de la propiedad.

Su aplicación puede motivarse en estrategias bioclimáticas, mejorar la aislación acústica y térmica, pero principalmente para aprovechar la naturaleza como herramienta de diseño, variable, viva y renovable.

La aplicación de muros verdes a escala urbana podría tener un impacto beneficioso en la mitigación de la isla de calor, la fijación del CO2, la preservación de la biodiversidad y la consolidación de la identidad arquitectónica local.

La mejora psicológica en la población, ya sea el propio usuario o el ciudadano común, es otro aspecto favorable, dado que el remplazo del gris por el verde disminuye el estrés asociado a la vida urbana.

\section{AGRADECIMIENTOS}

A la Arq. Alejandra Maro, coautora de la Torre Vista, que brindó precisiones sobre la implementación del muro verde, su impacto vivencial en el edificio y los costos de mantenimiento.

Al Arq. Gustavo Barrios D' Ambra, autor del Consejo Profesional, que brindó su tiempo y la totalidad de la documentación técnica del proyecto, fotos del proceso constructivo y de la obra terminada.

Al personal del Centro Biotecnológico Forestal, especialmente al Lic. en Biotecnología Pablo Klusacek.

\section{BIBLIOGRAFÍA}

Benyus, J. (2012). Biomimesis. Cómo la ciencia innova inspirándose en la naturaleza. Barcelona: Tusquets Editores S.A.

Braungart, M. y Mcdonough, W. (2005). Cradle to cradle. Rediseñando la forma en que hacemos las cosas. Madrid: McGraw Hill.

D’Elia, María y Pilar, Claudia (2018). Análisis ambiental de muros verdes para las condiciones de la Región NEA. Informe Final de Beca CIN, Corrientes, Universidad Nacional del Nordeste.

Evans, J. (2010). Sustentabilidad en Arquitectura. Consejo Profesional de Arquitectura y Urbanismo.

IRAM 11601. (1996). Acondicionamiento Térmico de Edificios. Métodos de Cálculo. Propiedades Térmicas de los componentes y elementos de construcción en régimen estacionario.

IRAM 11603. (1996). Acondicionamiento Térmico de Edificios. Clasificación Bioambiental de la República Argentina.

IRAM 11605. (2006). Acondicionamiento Térmico de Edificios. Condiciones de Habitabilidad en Edificios. Valores máximos de transmitancia térmica en cerramientos opacos.

IRAM 11930. (2010). Construcción Sostenible. Principios Generales. Buenos Aires: Instituto Argentino de Normalización y Certificación.

MARQ estudio. Disponible en http://www.estudiomarq.com.ar

Navarro Portilla, J. (2013). Los jardines verticales en la edificación. Trabajo final de máster en edificación, Escuela Técnica Superior Ingeniería de Edificación, Universidad Politécnica de Valencia.

Zurigarín, G. (Ed). (2013) EA. Envolventes arquitectónicas. Editorial EA. Disponible en http://www.envolvente-arquitectonica.com/revistas/06/EA06.pdf 